\title{
CoolBox: a flexible toolkit for visual analysis of genomics data
}

\author{
Weize Xu ${ }^{1,2}$, Quan Zhong ${ }^{3}$, Da Lin ${ }^{1,2}, Y_{a} Z_{\text {Zuo }}{ }^{1}$, Jinxia Dai ${ }^{1,2}$, Guoliang Li $\mathrm{Li}^{3,4,5,6^{*}}$ and Gang Cao ${ }^{1,2,3,4^{*}}$
}

\author{
${ }^{*}$ Correspondence: \\ guoliang.li@mail.hzau.edu.cn; \\ gcao@mail.hzau.edu.cn \\ ${ }^{4}$ College of Bio-Medicine \\ and Health, Huazhong \\ Agricultural University, \\ Wuhan, China ${ }^{6}$ Hubei Key \\ Laboratory of Agricultural \\ Bioinformatics, Hubei \\ Engineering Technology \\ Research Center \\ of Agricultural Big Data, 3D \\ Genomics Research Center, \\ Huazhong Agricultural \\ University, Wuhan, China \\ Full list of author information \\ is available at the end of the \\ article
}

\begin{abstract}
Background: Data visualization, especially the genome track plots, is crucial for genomics researchers to discover patterns in large-scale sequencing dataset. Although existing tools works well for producing a normal view of the input data, they are not convenient when users want to create customized data representations. Such gap between the visualization and data processing, prevents the users to uncover more hidden structure of the dataset.
\end{abstract}

Results: We developed CoolBox - an open-source toolkit for visual analysis of genomics data. This user-friendly toolkit is highly compatible with the Python ecosystem and customizable with a well-designed user interface. It can be used in various visualization situations like a Swiss army knife. For example, to produce high-quality genome track plots or fetch commonly used genomic data files with a Python script or command line, to explore genomic data interactively within Jupyter environment or web browser. Moreover, owing to the highly extensible Application Programming Interface design, users can customize their own tracks without difficulty, which greatly facilitate analytical, comparative genomic data visualization tasks.

Conclusions: CoolBox allows users to produce high-quality visualization plots and explore their data in a flexible, programmable and user-friendly way.

Keywords: Genomics, Visualization, Genome browser

\section{Background}

With the rapid development of Next-Generation Sequencing (NGS) technologies, more and more genomic assays have been developed to profile the genome from various aspects, such as RNA expression [1], protein-DNA binding [2], chromatin accessibility [3] and 3D structure [4, 5]. By integrating data from such types of different assays or the so-called multi-omics approach, biologists can comprehensively investigate genome dynamics during biological processes. This methodology has been successfully applied to many biological fields, such as neurological diseases [6], development of nervous system [7] and virus infection [8,9]. Data visualization, especially the genome track like plots, are crucial for exploring or demonstrating some local or global properties of the genomics data. author(s) and the source, provide a link to the Creative Commons licence, and indicate if changes were made. The images or other third party material in this article are included in the article's Creative Commons licence, unless indicated otherwise in a credit line to the material. If material is not included in the article's Creative Commons licence and your intended use is not permitted by statutory regulation or exceeds the permitted use, you will need to obtain permission directly from the copyright holder. To view a copy of this licence, visit http:// creativecommons.org/licenses/by/4.0/. The Creative Commons Public Domain Dedication waiver (http://creativecommons.org/publicdomain/zero/1.0/) applies to the data made available in this article, unless otherwise stated in a credit line to the data. 
Many visualization tools have been developed to meet these demands, and these tools can be classified into three categories: (1) Command-line plotting tool [10, 11], (2) Graphical User Interface(GUI) software [12], and (3) Web-based track browser [13-15]. In different situations, each kind of tools has its own advantages and limitations. As for command-line tools, they are convenient for bioinformaticians to produce plots or results easily but require Linux command line skills. GUI tools are friendly to people who are not skilled at programming and command line. Web-based browsers could share visualization results between colleagues. However, they are not efficient in transmission and have relative high latency between the websites and customers. Moreover, for program developers, GUI and web-based tools are not as convenient as command-line tools and plotting packages, which could be locally installed and easily called between stacks. Despite the above tools work well for providing an overview of the input genomic data. However, during actual scientific research, users need a detail comparative and analytical data visualization more than just the basic view of the data. For example, to visualize the differential contact interaction (DCI) of two Hi-C contact matrices [16] or predicted chromatin loops on the matrix [17]. In most cases, bioinformaticians work in programmatic and interactive environments like RStudio, IPython console and Jupyter notebook to complete the data analysis, algorithm development and visualization tasks. However, there is a gap between the data analysis ecosystem and the existing genomic data visualization tools. Researchers spend a lot of time on unnecessary stuffs like file format conversion and environment switching. Therefore, a versatile tool that fills the gap will significantly facilitate the genomics study.

To fill this gap, we developed CoolBox, a versatile toolkit for exploration-driven visualization of genomic data. It combines advantages of existing tools and is highly compatible with the Python scientific ecosystem, highly customizable, easy to use with intuitive interface design and simple installation procedure. It can be used in different scenarios: (1) Python script or another python package for data fetching and plotting; (2) Shell as a commandline plotting tool; (3) Jupyter notebook environment for data fetching, plotting, and exploration; and (4) Web application for exploration and demonstration within the web browser.

\section{Implementation}

The plotting system of CoolBox is based on the matplotlib package. A part of the plotting code in the CoolBox is a fork from pyGenomeTracks package. [10] The data stored in bigWig, "cool" and "hic" file format are loaded through pybbi (https:/github.com/nvictus/ pybbi), cooler [18] and straw [19] packages. Pairwise interaction data in Browser Extensible Data Paired-End (BEDPE) and Pairs format is indexed and randomly accessed using the pairix software (https://github.com/4dn-dcic/pairix). Other text-based genomic feature data format, like Browser Extensible Data (BED), Gene transfer format (GTF), and BedGraph is indexed and random accessed using the tabix [20] software. The widget panel in the GUI is implemented by using the ipywidgets package.

\section{Results and discussion}

\section{Flexible and user-friendly API and CLI for producing high-quality genome track plots}

CoolBox provides an Application Programming Interface (API) for Python script or Jupyter environment as well as a Command Line Interface (CLI) for Shell. The interface design is inspired by the popular R package ggplot2 [21]. It allows users to compose 
their figures with highly intuitive syntax. In CoolBox, users can use the "+" operator in Python or "add" command in Shell to compose low-level track elements to a higherlevel figure. For example, to compose track objects of various kinds of genomic data into a single frame and interactively review interested regions in genome browser with few lines of Python codes or Shell commands (Fig. 1).

Besides the 1-dimensional viewing mode supported by most other visualization tools, CoolBox supports a joint-view mode that enables users to visualize trans or cis-remote regions in a Hi-C contact matrix (Fig. 2).

Most sets of commonly generated genomic assay data such as RNA-Seq, ChIP-Seq, ATAC-Seq, Hi-C, HiChIP [22] data which stored in BedGraph, bigwig [23], cool [18], .hic [19] and other file formats (see Table 1) can be visualized in CoolBox by different kinds of tracks. Most tracks' features (color, height, style, etc.) can also be configured in the same way via the API or CLI. In the CoolBox plotting system, the plot contains not only a single layer. Users can put another layer (Coverage) upon the original plot to produce more comprehensive and high-quality figures. Furthermore, figures can be generated in different formats, including PNG, JPEG, PDF, and SVG. More details about the API and CLI are available in the online documents and user manual.

\section{Interactive exploration and reproducible analysis on genomic data}

As shown in Fig. 3, CoolBox provides a GUI for interactive data visualization, by which users can explore different genomic regions by operating a simple widget panel and visualize the data within a specific region.

Besides, the data and the figures are bound together by Python objects. In this way, users can get the precise data of each track within the current view of the genomic region through the API. This design facilitates comparative visualization and statistical analysis. CoolBox is also a general genomic-file reading package. Data within a particular

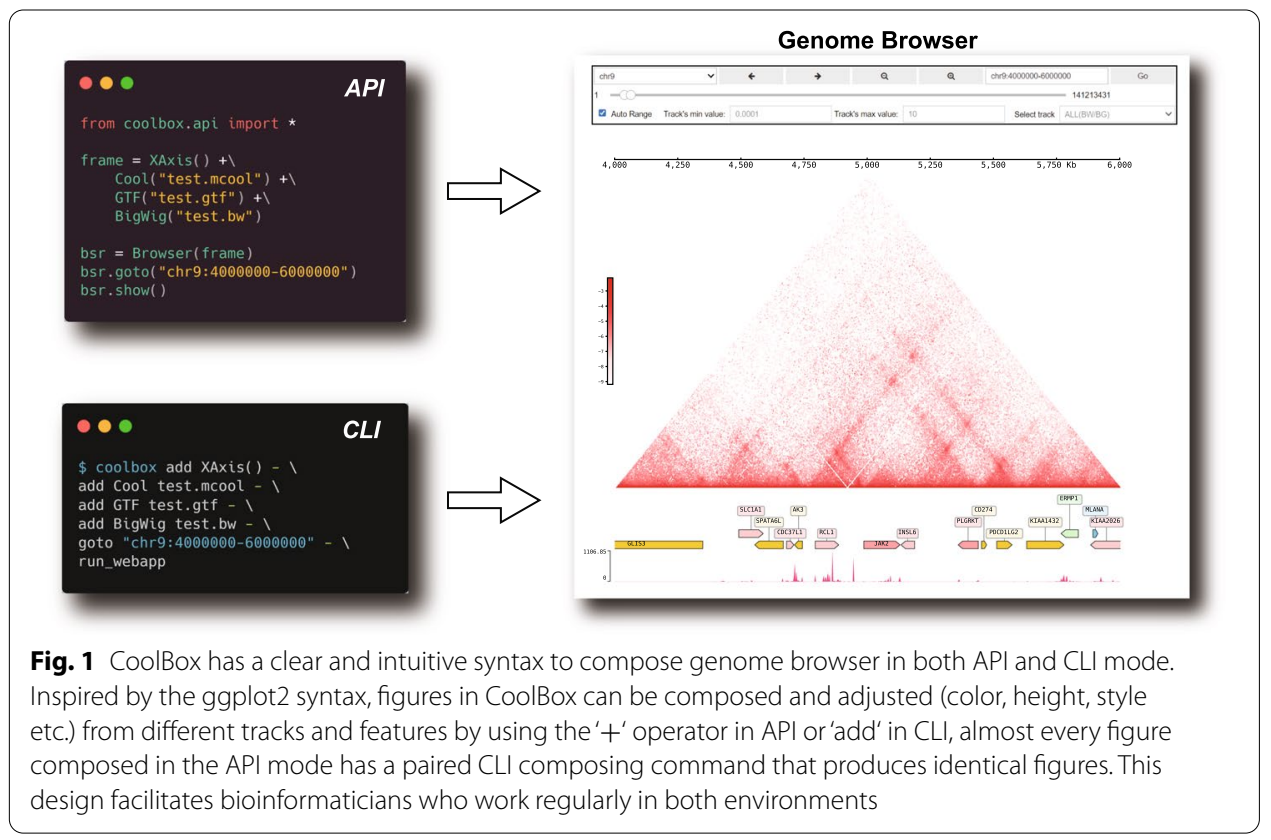


(A)

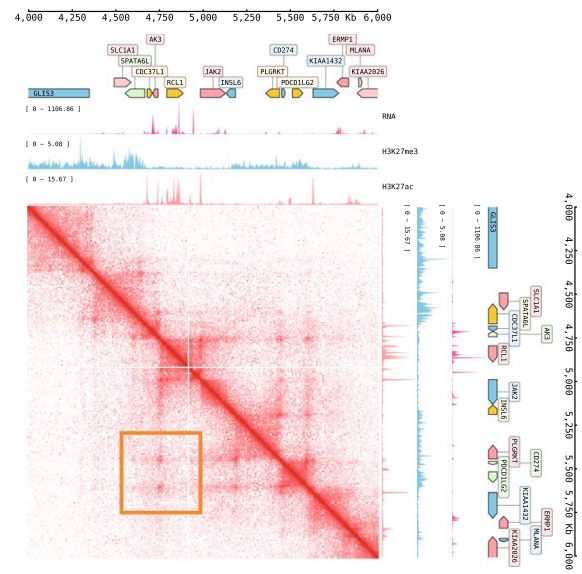

(B)

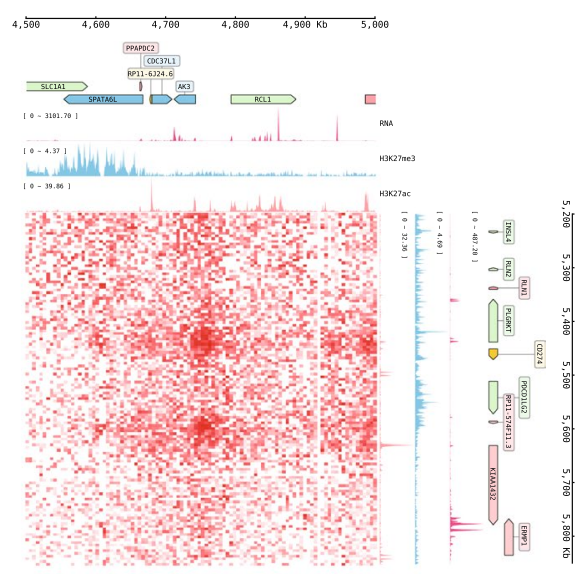

Fig. 2 Joint (2d) view example, CoolBox can compose big figure which put frames around a center contact matrix. This allows to visualize the trans or cis remote (off-diagonal) contact matrix along with genome features. A Joint view on an on-diagonal region. B Joint view on an cis remote region, which shows the magnified detail of the orange box marked loop region that contains two chromatin loops in (A)

Table 1 A part of CoolBox builtin tracks for visualizing different kinds of genomics data formats

\begin{tabular}{lll}
\hline Track type & File format & Description \\
\hline XAxis & None & Coordinate of the reference genome \\
Spacer & None & For add vertical space between two tracks \\
BigWig & .bigwig & Track for bigWig file, draw the histogram \\
BedGraph & .bedgraph & Track for BedGraph file, draw the histogram \\
BAM & .bam & BAM track for visualize the coverage or alignment \\
BED & .bed & For visualization genome annotation, like refSeq genes and chromatin states \\
GTF & .gtf & Track of GTF file, for visualize gene annotation \\
Arcs & .pairs, .bedpe & Show the chromosome interactions get from ChIA-PET, HiChIP or Hi-C loop data \\
HiCMat & .cool, .mcool, .hic & Show the chromosome contact matrix from Hi-C data \\
Virtual4C & .cool, .mcool, .hic & Virtual 4C track, using Hi-C data to mimic 4C \\
DiScore & .cool, .mcool, .hic & Directional index of Hi-C matrix for detecting TAD \\
InsuScore & .cool, .mcool, .hic & Insulation score of Hi-C matrix for inferring TAD borders \\
HiCDiff & .cool, .mcool, .hic & Show the difference between two contact matrix \\
Selfish & .cool, .mcool, .hic & $\begin{array}{l}\text { Apply the selfish algorithm [16] on two contact matrices to detect differential } \\
\text { contact interactions }\end{array}$ \\
SNP & .tsv & Track for show SNPs Manhattan plot \\
\hline
\end{tabular}

genome region can be retrieved in a short time, as almost all supported file formats can be indexed and randomly accessed.

Moreover, by leveraging the power of the Jupyter notebook, the visualization result and the entire process can be recorded in the notebook. It is convenient for sharing the visualization result and reproducing the whole analysis by other researchers.

\section{A testing and visualizing framework for new algorithm development}

Owing to the user-friendly and highly extensible API design, users can implement their custom tracks without any difficulty, thus enabling seamless cooperation in Python-based algorithm development and scientific research. The algorithm 


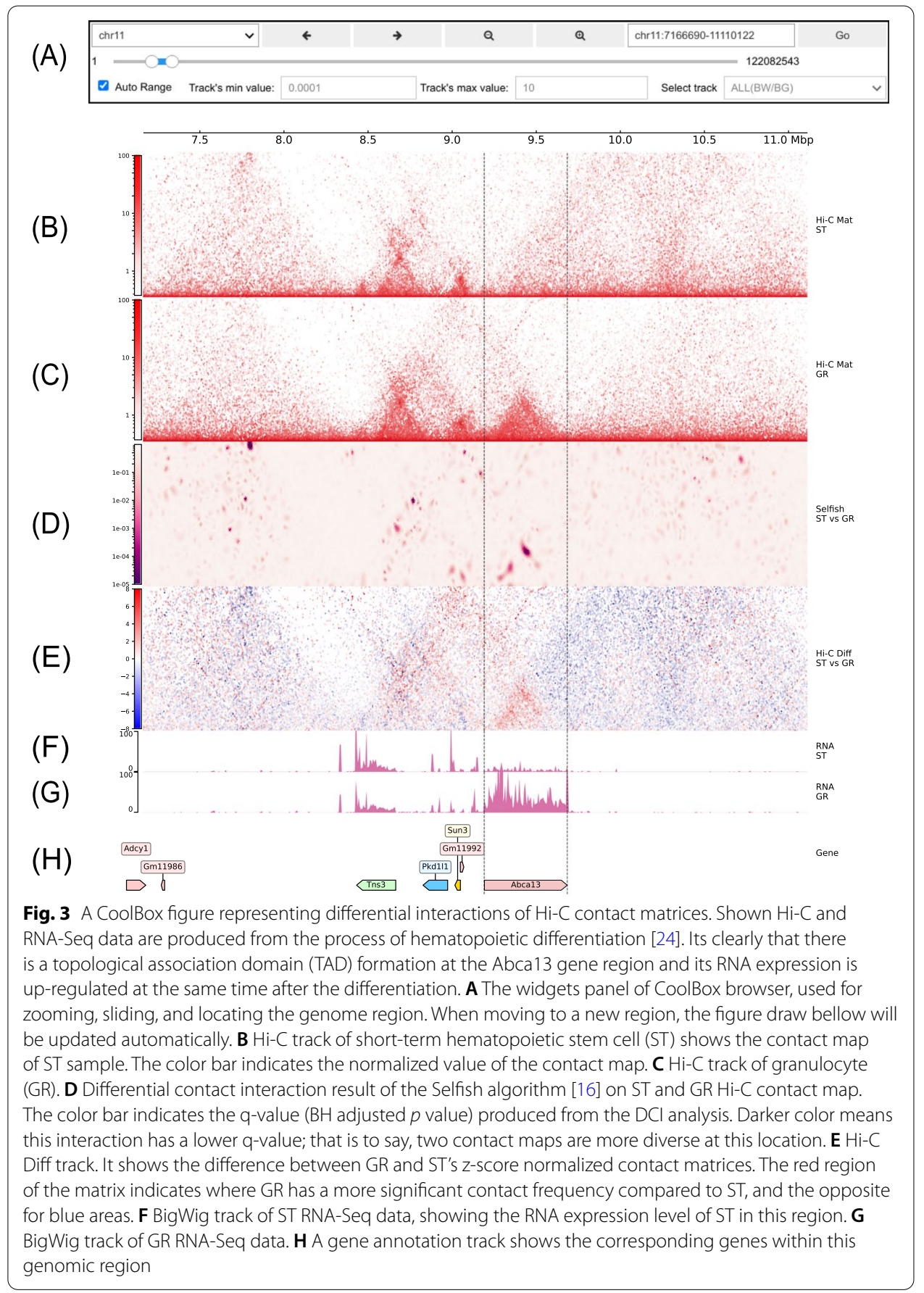

developer can check and visualize the intermediate results produced by their algorithm and adjust parameters simultaneously. In addition, as CoolBox uses an objectoriented programming paradigm in its design, users can reuse each track's codes by inheritance, including data extraction and drawing-related functions. In most cases, users only need to write algorithm-related core parts. The most tedious part including raw-data reading, preprocessing, and figure drawing are handed over to CoolBox through inheritance (see method section and user manual for implementation 
details). In this way, bioinformaticians can free themselves from those repetitive procedures and only focuses on the data post-processing.

We demonstrate the advantages by implementing a track that visualizes the outputs of the Peakachu algorithm [17], which is a RandomForest based method for detecting loops in the Hi-C contact matrix. As depicted in Fig. 4, the main part of the whole track contains merely 20 lines of Python code. The data fetching and plotting functionality are fully reused by inheriting Cool/ArcsBase Track base class. Furthermore, the customdefined track is empowered to be used in CLI, API, and browser mode in couple with other built-in tracks. More details include a reproducible code block and can be found in the online documents and user manual.

\section{Comparison with other existing visualization tools}

As stated before, there is an urgent need for better visualization tools to accelerate the integration and mining of biological data. Therefore, more and more visualization tools have been developed in recent years. A comparison of features between CoolBox and these tools is listed in Table 2. Most of the visualization tools require a tedious installation process and are operated through the command line. Before visualization, the data needs to be preprocessed through specific steps, and then a static or interactive web interface is generated.

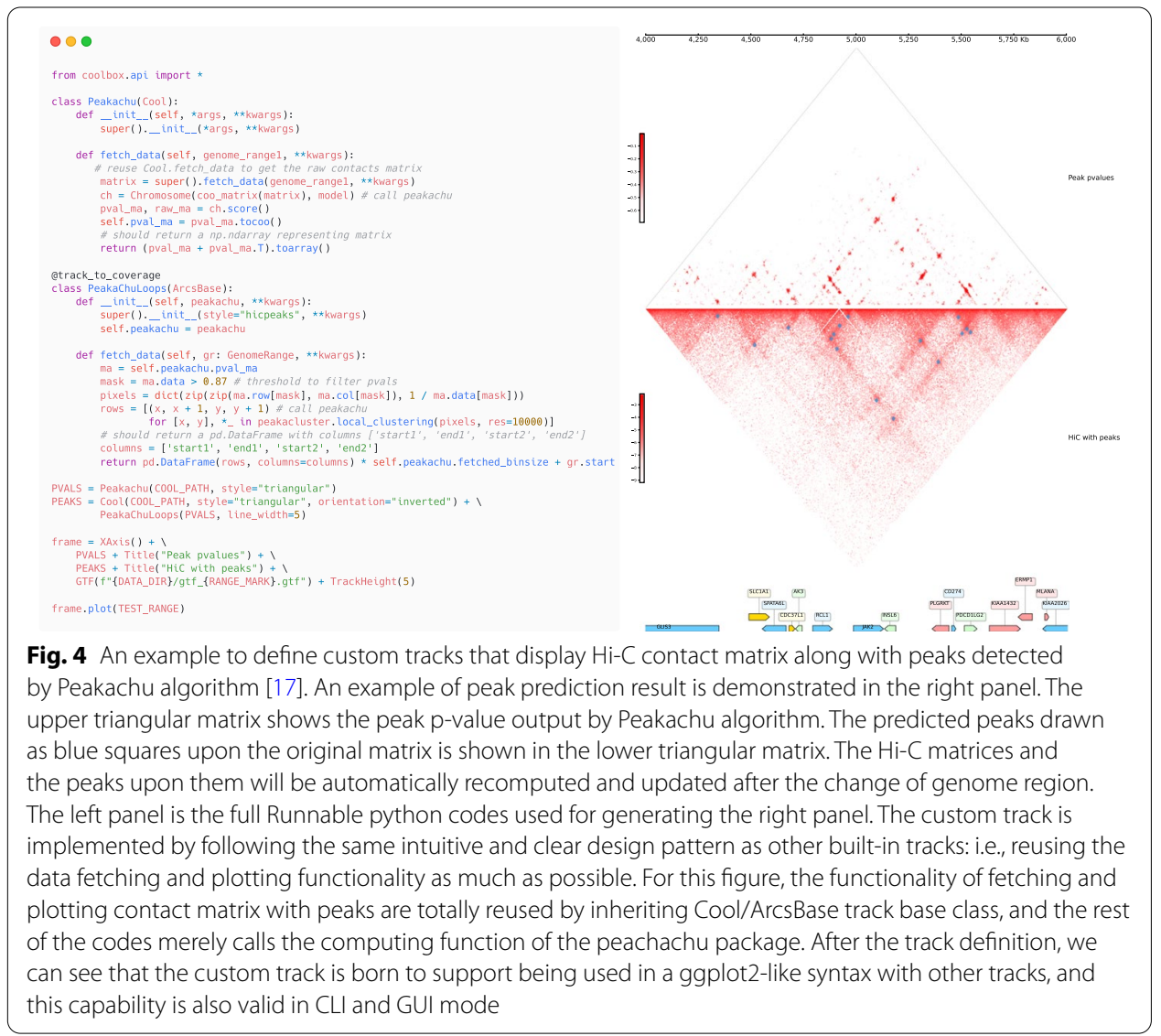


The visualization and data processing of most visualization tools are dissociated, which is not convenient for bioinformaticians whose routine works rely on Pythonbased scientific computation ecosystem. Except for the CLI mode supported by most visualization tools, the API that the CoolBox has been used internally and exposed follows the same design as the CLI, making switching between these two modes with no pain. More importantly, since the API in CoolBox combines computation and visualization, users can dynamically add different tracks or even custom tracks in the python notebook while processing raw data or developing new methods.

\section{Conclusion}

CoolBox is a versatile toolkit for the visualization and exploration of multi-omics data in the Python ecosystem. It provides a user-friendly ggplot2-like syntax for composing various kinds of tracks in CLI, API, GUI and web browser mode. More importantly, its built on a highly extensible plotting system that allows users to implement their custom tracks without wasting time on data fetching and figure plotting procedures. Through the power of Jupyter notebook, it provides a convenient way for bioinformaticians to exploit it's versatility for better personalized data manipulation and demonstration. It could also increase the reproducibility of genomic data visualization tasks as codes and figures are all organized into the same page.

Table 2 Summary of genomic visualization tools

\begin{tabular}{|c|c|c|c|c|}
\hline Tools & $\begin{array}{l}\text { Programming } \\
\text { language }\end{array}$ & API plot & CLI plot & Online access \\
\hline CoolBox & Python & $\checkmark$ & $\checkmark$ & $\checkmark$ \\
\hline pyGenomeTracks & Python & & $\checkmark$ & \\
\hline gcMapExplorer & Python & $\checkmark$ & & \\
\hline HiCPlotter & Python & & $\checkmark$ & \\
\hline HiGlass & Python, HTML, CSS, JS & $\checkmark$ & & $\checkmark$ \\
\hline YueLab Browser & HTML, CSS, JS & & & $\checkmark$ \\
\hline WashU Browser & HTML, CSS, JS & & & $\checkmark$ \\
\hline TADkit & HTML, CSS, JS & & & $\checkmark$ \\
\hline JuiceBox.js & HTML, CSS, JS & & & $\checkmark$ \\
\hline JuiceBox & Java & & & \\
\hline$\overline{\mathrm{GUI}}$ & Input & Installation & Customization & \\
\hline \multirow[t]{2}{*}{ Web and Jupyter } & Raw data & Bioconda or PyPI & Python knowledge, very easy & \\
\hline & Raw data & PyPI & Python knowledge, easy & \\
\hline \multirow[t]{2}{*}{ Local } & Preprocessed data & PyPI & Python knowledge, easy & \\
\hline & Preprocessed data & Manually install & & \\
\hline Web and Jupyter & $\begin{array}{l}\text { Preprocessed data, } \\
\text { via network }\end{array}$ & Docker & Web knowledge & \\
\hline Web & Via network & & & \\
\hline Web & Via network & & & \\
\hline Web & $\begin{array}{l}\text { Preprocessed data, } \\
\text { via network }\end{array}$ & Manually install & & \\
\hline Web & Via network & & & \\
\hline Local & Raw data & Download & & \\
\hline
\end{tabular}




\title{
Availability and requirements
}

\author{
Project name: CoolBox
}

Project home page: https:/github.com/GangCaoLab/CoolBox

Operating system(s): Linux, macOS, Windows WSL

Programming language: Python

Other requirements: All software requirements are listed in https:/github.com/

GangCaoLab/CoolBox/blob/master/environment.yml

License: GPLv3

Any restrictions to use by non-academics: GPLv3 licensing restrictions apply.

\section{Abbreviations}

NGS: Next-generation sequencing; GUI: Graphical user interface; API: Application programming interface; CLI: Command line interface.

\section{Acknowledgements}

We thank everyone who contributed to this project on GitHub. We thank Khaista Rahman for helping to review and improve our English writing.

\section{Authors' contributions}

WX: Conceptualization, Investigation, Software design, Software maintain, Software test, Writing-Original Draft Preparation, Writing-Review and Editing. QZ: Conceptualization, Software design, Software maintain, Software test, WritingOriginal Draft Preparation, Writing—Review and Editing. DL: Conceptualization, Resources, Writing—Review and Editing. YZ: Conceptualization, Writing — Review and Editing. JD: Conceptualization, Funding Acquisition, Supervision, Writing Review and Editing. LG: Conceptualization, Funding Acquisition, Investigation, Resources, Supervision, Writing-Review and Editing. GC: Conceptualization, Funding Acquisition, Investigation, Resources, Supervision, Writing-Review and Editing. All the authors have read and approved the final manuscript.

\section{Funding}

1. The Key Research and Development Program of Guangdong Province (Grant $2019 B 020211003$ to Gang Cao). The funders had no role in study design, data collection and analysis, decision to publish, or preparation of the manuscript. 2. National Natural Science Foundation of China (NSFC) (Grants 31941014 to Gang Cao). The funders had no role in study design, data collection and analysis, decision to publish, or preparation of the manuscript. 3. National Natural Science Foundation of China (NSFC) (Grants 31941005 to Huanchun Chen). The funders had no role in study design, data collection and analysis, decision to publish, or preparation of the manuscript. 4. National Natural Science Foundation of China (Grants 31702196 to Ke Xiao). The funders had no role in study design, data collection and analysis, decision to publish, or preparation of the manuscript. 5. China Postdoctoral Science Foundation (Grant 2019M662676, to Ke Xiao). The funders had no role in study design, data collection and analysis, decision to publish, or preparation of the manuscript.

Availability of data and materials

Sample data designed to demonstrate most features of the software is provided at https://github.com/GangCaoLab/ CoolBox/tree/master/tests/test_data.

\section{Declarations}

Ethics approval and consent to participate

Not applicable.

\section{Consent for publication}

Not applicable.

\section{Competing interests}

The authors declare that they have no competing interests.

\section{Author details}

${ }^{1}$ College of Veterinary Medicine, Huazhong Agricultural University, Wuhan, China. ${ }^{2}$ State Key Laboratory of Agricultural Microbiology, Huazhong Agricultural University, Wuhan, China. ${ }^{3}$ College of Informatics, Huazhong Agricultural University, Wuhan, China. ${ }^{4}$ College of Bio-Medicine and Health, Huazhong Agricultural University, Wuhan, China. ${ }^{5}$ National Key Laboratory of Crop Genetic Improvement, Huazhong Agricultural University, Wuhan, China. ${ }^{6}$ Hubei Key Laboratory of Agricultural Bioinformatics, Hubei Engineering Technology Research Center of Agricultural Big Data, 3D Genomics Research Center, Huazhong Agricultural University, Wuhan, China. 
Received: 2 June 2021 Accepted: 27 September 2021

Published online: 10 October 2021

\section{References}

1. Morin RD, Bainbridge M, Fejes A, Hirst M, Krzywinski M, Pugh TJ, McDonald H, Varhol R, Jones SJ, Marra MA. Profiling the HeLa s3 transcriptome using randomly primed cDNA and massively parallel short-read sequencing. Biotechniques. 2008;45(1):81-94.

2. Robertson G, Hirst M, Bainbridge M, Bilenky M, Zhao Y, Zeng T, Euskirchen G, Bernier B, Varhol R, Delaney A, et al. Genome-wide profiles of stat1 DNA association using chromatin immunoprecipitation and massively parallel sequencing. Nat Methods. 2007;4(8):651.

3. Buenrostro JD, Giresi PG, Zaba LC, Chang HY, GreenleafWJ. Transposition of native chromatin for multimodal regulatory analysis and personal epigenomics. Nat Methods. 2013;10(12):1213.

4. Lieberman-Aiden E, Van Berkum NL, Williams L, Imakaev M, Ragoczy T, Telling A, Amit I, Lajoie BR, Sabo PJ, Dorschner $\mathrm{MO}$, et al. Comprehensive mapping of long-range interactions reveals folding principles of the human genome. Science. 2009;326(5950):289-93.

5. Fullwood MJ, Ruan Y. Chip-based methods for the identification of long-range chromatin interactions. J Cell Biochem. 2009:107(1):30-9.

6. Corces MR, Shcherbina A, Kundu S, Gloudemans MJ, Frésard L, Granja JM, Louie BH, Eulalio T, Shams S, Bagdatli ST, et al. Single-cell epigenomic analyses implicate candidate causal variants at inherited risk loci for Alzheimer's and Parkinson's diseases. Nat Genet. 2020;52(11):1158-68.

7. Song M, Pebworth M-P, Yang X, Abnousi A, Fan C, Wen J, Rosen JD, Choudhary MN, Cui X, Jones IR, et al. Cell-typespecific 3d epigenomes in the developing human cortex. Nature. 2020;587(7835):644-9.

8. Heinz S, Texari L, Hayes MG, Urbanowski M, Chang MW, Givarkes N, Rialdi A, White KM, Albrecht RA, Pache L, et al. Transcription elongation can affect genome 3d structure. Cell. 2018;174(6):1522-36.

9. Cao C, Hong P, Huang X, Lin D, Cao G, Wang L, Feng B, Wu P, Shen H, Xu Q, et al. HPV-CCDC106 integration alters local chromosome architecture and hijacks an enhancer by three-dimensional genome structure remodeling in cervical cancer. J Genet Genomics. 2020;47(8):437-50.

10. Lopez-Delisle L, Rabbani L, Wolff J, Bhardwaj V, Backofen R, Grüning B, Ramírez F, Manke T. pyGenome Tracks: reproducible plots for multivariate genomic data sets. Bioinformatics. 2020;37:422.

11. Akdemir KC, Chin L. Hicplotter integrates genomic data with interaction matrices. Genome Biol. 2015;16(1):198.

12. Kumar R, Sobhy H, Stenberg P, Lizana L. Genome contact map explorer: a platform for the comparison, interactive visualization and analysis of genome contact maps. Nucleic Acids Res. 2017;45(17):152

13. Wang Y, Song F, Zhang B, Zhang L, Xu J, Kuang D, Li D, Choudhary MN, Li Y, Hu M, et al. The 3d genome browser: a web-based browser for visualizing $3 \mathrm{~d}$ genome organization and long-range chromatin interactions. Genome Biol. 2018;19(1):1-12

14. Li D, Hsu S, Purushotham D, Sears RL, Wang T. Washu epigenome browser update 2019. Nucleic Acids Res. 2019:47(W1):158-65.

15. Kerpedjiev P, Abdennur N, Lekschas F, McCallum C, Dinkla K, Strobelt H, Luber JM, Ouellette SB, Azhir A, Kumar N, et al. Higlass: web-based visual exploration and analysis of genome interaction maps. Genome Biol. 2018;19(1):1-12

16. Ardakany AR, Ay F, Lonardi S. Selfish: discovery of differential chromatin interactions via a self-similarity measure. Bioinformatics. 2019;35(14):145-53.

17. Salameh TJ, Wang X, Song F, Zhang B, Wright SM, Khunsriraksakul C, Ruan Y, Yue F. A supervised learning framework for chromatin loop detection in genome-wide contact maps. Nat Commun. 2020;11(1):1-12.

18. Abdennur N, Mirny LA. Cooler: scalable storage for $\mathrm{Hi}-\mathrm{C}$ data and other genomically labeled arrays. Bioinformatics. 2019;36:311-6. https://doi.org/10.1093/bioinformatics/btz540.

19. Durand NC, Shamim MS, Machol I, Rao SS, Huntley MH, Lander ES, Aiden EL. Juicer provides a one-click system for analyzing loop-resolution Hi-C experiments. Cell Syst. 2016;3(1):95-8.

20. Li H. Tabix: fast retrieval of sequence features from generic tab-delimited files. Bioinformatics. 2011;27(5):718-9.

21. Wickham H. ggplot2: elegant graphics for data analysis. J Stat Softw. 2010;35(1):65-88.

22. Mumbach MR, Rubin AJ, Flynn RA, Dai C, Khavari PA, Greenleaf WJ, Chang HY. Hichip: efficient and sensitive analysis of protein-directed genome architecture. Nat Methods. 2016;13(11):919-22.

23. Kent WJ, Zweig AS, Barber G, Hinrichs AS, Karolchik D. Bigwig and bigbed: enabling browsing of large distributed datasets. Bioinformatics. 2010;26(17):2204-7.

24. Zhang C, Xu Z, Yang S, Sun G, Jia L, Zheng Z, Gu Q, Tao W, Cheng T, Li C, et al. tagHi-C reveals 3d chromatin architecture dynamics during mouse hematopoiesis. Cell Rep. 2020;32(13):108206.

\section{Publisher's Note}

Springer Nature remains neutral with regard to jurisdictional claims in published maps and institutional affiliations. 